\title{
Analyse de métaux traces dans les produits pétroliers, état de l'art
}

\author{
C.P. Lienemann ${ }^{1}$ \\ 1 IFP-Lyon, Laboratoire AAS-ICP-FX, Direction Physique et Analyse, 69390 Vernaison - France \\ e-mail : charles.lienemann@ifp.fr
}

Résumé - Après une brève introduction sur la problématique des métaux traces dans l'industrie pétrolière, un retour sur les trois techniques majeures de dosages des métaux traces dans les produits pétroliers est présenté reprenant les travaux effectués dans les dix dernières années. Ainsi les différents types d'introduction, utilisés pour la spectrométrie d'absorption atomique avec four électrothermique (GFAAS), la spectrométrie d'émission atomique par plasma à couplage inductif (ICP-OES) et la spectrométrie de masse par plasma à couplage inductif (ICP-MS), sont repris et comparés en terme de limite de détection atteinte. Les applications sont diverses et touchent la totalité des produits pétroliers, du biocarburant en passant par l'éthanol, aux plus classiques essences et gazoles, allant jusqu'aux produits plus lourds que sont les bitumes. Une revue sur les principaux couplages possibles permet d'entrevoir les différentes possibilités que ces techniques pourront offrir dans les années à venir en terme de spéciation des métaux dans les produits pétroliers.

\begin{abstract}
Analysis of Trace Metals in Petroleum Products, State of the Art-Following a brief introduction to the problems posed by trace metals in the petroleum industry, a review of the three main techniques available for trace metals determination is presented, with their recent developments. Different introduction modes, used for graphite furnace atomic absorption spectrometry (GFAAS), inductively coupled plasma with optical emission spectrometry and inductively coupled plasma with mass spectrometry, are discussed and compared in terms of detection limits. Applications are various and apply to all kinds of petroleum products, from bioethanol to the more common gasoline and diesel, and also to heavier products such as bitumen. A review of the main chromatographic techniques coupled with ICP demonstrates the promise of these techniques for the future in terms of speciation of trace metals in petroleum products.
\end{abstract}




\section{GLOSSAIRE}

ETV

DIHEN

DI-ICP-MS

DIN

GC-ICP-MS

GFAAS

HPLC-ICP-MS

ICP-HRMS

ICP-MS

ICP-OES

ICP-QMS

LA-ICP-MS/OES Spectrométrie d'émission atomique/ de

LOD

$\mathbf{M} / \mathbf{Z}$

NIST

USN

UV masse par plasma à couplage inductif couplée à ICP.

Electro-Thermal Vaporization, vaporisation électrothermale utilisée lorsque l'on couple un four graphite à une détection ICP-MS.

Direct Injection High Efficiency Nebulizer, nébuliseur à injection directe haute efficacité.

Dilution Isotopique - Spectrométrie de masse par plasma à couplage inductif.

Direct Injection Nebulizer, nébuliseur à injection directe.

Spectrométrie de masse par plasma à couplage inductif couplé avec la chromatographie en phase gazeuse.

Graphite Furnace Atomic Absorption Spectrometry, Spectrométrie d'Absorption Atomique avec Four Graphite.

Spectrométrie de masse par plasma à couplage inductif couplé avec la chromatographie en phase liquide.

Inductively Coupled Plasma-High Resolution Mass Spectrometry, Spectrométrie de masse haute résolution par plasma à couplage inductif.

Inductively Coupled Plasma-Mass Spectrometry, Spectrométrie de masse par plasma à couplage inductif.

Inductively Coupled Plasma-Optical Emission Spectroscopy, Spectrométrie d'émission atomique par plasma à couplage inductif.

Inductively Coupled Plasma-Quadrupolar Mass Spectrometry, Spectrométrie de masse quadrupolaire par plasma à couplage inductif.

Limit Of Detection, limite de détection.

Ratio masse (M) sur charge (Z) utilisé en spectrométrie de masse pour la séparation des ions.

National Institute of Standard and Technology, organisme américain fournisseur de matériaux de référence.

UltraSonic Nebulizer, Nébuliseur ultrasonique.

Ultra-Violet, région des longueurs d'onde entre 120 et $200 \mathrm{~nm}$ environ.

\section{INTRODUCTION}

L'existence de métaux dans les combustibles fossiles, et notamment dans les pétroles, fut établie dans les années 1930. Cependant, l'analyse des métaux dans les produits pétroliers pose de nombreux problèmes tant en terme de nature du produit qui peut être très diverse, que de la concentration des métaux, qui hormis le $\mathrm{Fe}$, $\mathrm{Ni}$ et $\mathrm{V}$ sont souvent en concentration faible et donc difficilement mesurables.

De même, les formes chimiques sous lesquelles les métaux dans les pétroles sont présents ainsi que leur distribution entre formes organiques et inorganiques sont souvent peu connues. Ceci est dû en particulier aux difficultés à analyser ces composés et ces éléments dans les pétroles.

D'un point de vue pétrochimique, l'analyse d'éléments traces métalliques dans les produits pétroliers peut servir à quantifier la présence dans les huiles d'éléments poisons pour les différents procédés de raffinage et de la conversion du pétrole. Ces éléments peuvent avoir été intégrés dans l'huile naturellement $(\mathrm{Hg}, \mathrm{As}, \mathrm{Ni}, \mathrm{V}$, etc.), par pollution (ex. : $\mathrm{Pb}$ ), ou dériver de la mise en œuvre de certains additifs (ex. : $\mathrm{Si}$ ). Ces effluents se doivent d'avoir des teneurs inférieures au $\mu \mathrm{g} / \mathrm{l}$. À ce jour, la plupart de ces pollutions est mise en évidence par une désactivation des catalyseurs de réaction, qui une fois déchargés et analysés montrent des teneurs cette fois-ci non négligeables en métaux provenant de l'accumulation dû au passage de plusieurs tonnes de produits pétroliers, ayant libérés les métaux sur les sites actifs du catalyseur.

L'industrie pétrolière à donc un besoin urgent de techniques rapides, sensibles et précises de ces différents éléments aux teneurs de l'ordre du ppb. Cet article reprend donc les avancées obtenues ces dix dernières années dans le dosage des métaux dans les produits pétroliers, avec essentiellement trois techniques que sont la spectrométrie d'absorption atomique avec four graphite électrothermique (GFAAS), la spectrométrie d'émission atomique par plasma à couplage inductif (ICP-OES) et la spectrométrie de masse par plasma à couplage inductif (ICP-MS).

\section{ÉTAT DE L'ART DES APPLICATIONS ACTUELLES POUR LES PRODUITS PÉTROLIERS}

\section{Spectrométrie d'absorption atomique à four graphite - Applications récentes sur produits pétroliers}

L'absorption atomique four (GFAAS) est la première technique ayant permis l'étude des métaux traces dans les produits pétroliers. Très répandue sur le globe par son prix relativement bas au regard des ICP-MS, adaptée aux concentrations mesurées de l'ordre de la dizaine de ppb, l'absorption atomique four est parmi les techniques les plus utilisées pour 
analyser les traces dans les milieux pétroliers. On retrouve donc dans la littérature portant sur la technique GFAAS la plupart des éléments mesurés en traces (As, Sb, Se, Ni, V, $\mathrm{Pb}, \mathrm{Cd}, \mathrm{Si}, \mathrm{Cu}, \mathrm{Mn}$, etc.). Les grands procédés de préparation d'échantillons propres à toutes les techniques de dosages en spectrométrie atomique (minéralisation, micro-émulsion, introduction directe) sont représentés en GFAAS avec une préférence forte ces dernières années pour les introductions sous forme organique ou sous forme de micro-émulsion.

L'injection organique directe est facilitée en GFAAS par le fait que celle-ci peut se faire à température ambiante suivie d'une montée en température qui peut être adaptée au type de produit pétrolier introduit. Ainsi une très bonne sensibilité est obtenue avec les naphtas et les essences qui sont habituellement très dilués avec les techniques utilisant une flamme (AAS classique) ou un plasma (ICP-OES et MS) pour des questions de volatilité du produit. En GFAAS en revanche, ces produits sont traités aisément puisque la fraction volatile est lentement éliminée par un programme de montée en température du four permettant l'élimination des composés organiques avant atomisation des éléments d'intérêt. L'avantage de cette méthode procédant par palier est même exploitée par Reboucas et al. (2003) qui procède par trois injections successives de $45 \mu \mathrm{l}$ de naphtas suivies de trois montées en température jusqu'à $180^{\circ} \mathrm{C}$ permettant d'éliminer les composés les plus volatiles (séchage) tout en concentrant dans le tube graphite les éléments d'intérêt avant atomisation. Cette technique permet de doser l'arsenic entre $0,5 \mu \mathrm{g} / \mathrm{l}$ et $120 \mu \mathrm{g} / \mathrm{l}$ avec $135 \mu \mathrm{l}$ de produit consommé. Le dosage effectué par Reboucas et al. (2003) sur onze produits pétroliers issus de l'Afrique et de l'Amérique du Sud démontre que le gain obtenu entre $5 \mu \mathrm{g} / \mathrm{l}$ et $0,5 \mu \mathrm{g} / \mathrm{l}$ permet de doser efficacement les onze produits contenant tous entre 0,6 et $2,9 \mu \mathrm{g} / \mathrm{l}$.

Plus classiquement, le dosage du vanadium dans les produits lourds est entrepris par Nakamoto et al. (2004) qui couvre ses tubes en graphite avec une solution de tungstène permettant de rallonger la vie des tubes et d'excéder les 200 injections. Aucune limite de quantification n'est donnée dans cet article et seule une validation de la méthode par un dosage de produit contenant entre 2 et $140 \mathrm{mg} / \mathrm{kg}$ est proposée.

L'avantage de pouvoir injecter des produits volatiles est repris dans les articles de Oliveira et al. (2002 a et b) qui dosent l'Al, $\mathrm{As}, \mathrm{Cu}, \mathrm{Fe}, \mathrm{Mn}$ et $\mathrm{Ni}$ dans de l'éthanol servant comme carburant alternatif. La préparation du tube graphite s'effectue par l'ajout de $250 \mu \mathrm{g}$ de $\mathrm{W}$ et de $250 \mu \mathrm{g}$ de $\mathrm{Rh}$ en temps que modificateur permanent. Une seule injection de $20 \mu \mathrm{l}$ d'un mélange 1:1 éthanol/acide nitrique complété par l'ajout d'une solution contenant $5 \mu \mathrm{g}$ de nitrate de $\mathrm{Pd}$ et de $3 \mu \mathrm{g}$ de nitrate de magnésium permet d'obtenir des limites de quantification de 2, 3, 0,6, 1, 0,4 et 1,3 $\mu \mathrm{g} / \mathrm{l}$ pour l'Al, As, $\mathrm{Cu}, \mathrm{Fe}, \mathrm{Mn}$ et Ni respectivement. Les modificateurs ajoutés permettent de maintenir le tube graphite en l'état pendant 700 injections. On observe donc dans le cas de l'As une meilleure limite de détection dans l'éthanol que dans le naphta à volume équivalent, ce qui est justifié par la complexité de la matrice pétrolière au regard de l'éthanol.

Plus récemment, Azeredo Amaro et Ferreira (2004) ont mis au point un dosage du Si dans les naphtas par absorption atomique four permettant de doser cet élément jusqu'à une limite de détection de $15 \mu \mathrm{g} / \mathrm{l}$. Au regard de la problématique d'empoisonnement par le Si des catalyseurs de l'industrie pétrolière, des essais infructueux en ICP-MS génés par la molécule $\mathrm{CO}^{+}$(masse $\mathrm{m} / \mathrm{Z}$ de 28 comme le $\mathrm{Si}$ ) généré par le plasma et empêchant la mesure de la masse $\mathrm{m} / \mathrm{Z}$ de $28 \mathrm{du} \mathrm{Si}$, l'intérêt de cette publication, où tous les paramètres instrumentaux sont décrits, semble évident. Une calibration linéaire entre 45 et $200 \mu \mathrm{g} / \mathrm{l}$ est effectuée avec un volume compris entre 10 et $30 \mu \mathrm{l}$. La répétabilité est de l'ordre de $10 \%$ pour une teneur à $50 \mathrm{ppb}$. Des mesures effectuées sur des échantillons de naphtas contenant entre 30 et $70 \mathrm{ppb}$ et dans lesquels des ajouts de 20 à 100 ppb ont été faits montrent des recouvrements toujours supérieurs à $95 \%$, ce qui s'avère être excellent à ces teneurs.

La plupart des travaux en GFAAS montrent qu'une partie importante du travail réside dans :

- la mise au point des conditions optimales de dépôt de l'échantillon dans le four graphite;

- la montée en température avant et pendant l'atomisation.

Cette mise au point est poussée dans son extrême avec Mbileni et al. (2002) où un four transverse poreux est utilisé pour injecter l'échantillon. La modification de la partie four de cet appareil n'est pas proposée dans le commerce mais semble pouvoir accepter des volumes d'injection plus important. Cette technologie semble même améliorer d'un facteur 4 les limites de quantification du dosage du $\mathrm{Pb}$ et de l'As à volume injecté identique vis-à-vis d'un appareil classique. Le gain obtenu avec la possibilité d'injecter un volume plus important n'est cependant pas donné dans cet article.

L'introduction de l'échantillon sous forme de micro-émulsion semble trouver des adeptes également dans le monde des utilisateurs de spectromètres d'absorption atomique en four graphite. Ainsi Aucelio et Curtius (2002) analyse l'As, le $\mathrm{Se}$ et $\mathrm{Sb}$ dans des essences et kérosènes à l'aide de ce mode d'introduction. Deux préparations ont été utilisées dans cette publication:

- un mélange de propanol, eau et essence ou kerosène sans surfactant;

- un mélange acide nitrique, le surfactant Triton X-100, eau et essence ou kérosène

avec dans les deux cas un ajout de $10 \% \mathrm{v} / \mathrm{v}$ de produits pétroliers vis-à-vis du volume final.

Les résultats finaux montrent que le mélange n'utilisant pas de surfactant n'est pas adapté à ce type de dosage et que dans le second cas l'injection de $10 \mu \mathrm{l}$ du mélange permet d'obtenir des limites de détection de 2, 3 et $4 \mu \mathrm{g} / \mathrm{l}$ pour l'As, le $\mathrm{Se}$ et $\mathrm{Sb}$ respectivement. 
Très récemment, le dosage $\mathrm{du} \mathrm{Cu}$, poison des catalyseurs Pd et Pt, a été entrepris par Dos Santos et al. (2005). Cette publication utilise également une introduction en microémulsion en mélangeant $3 \mathrm{ml}$ de condensats avec $0,6 \mathrm{ml}$ d'acide nitrique et $6,4 \mathrm{ml}$ de propanol. L'utilisation d'un surfactant ne semble pas nécessaire, mais la calibration doit impérativement être faite avec des étalons organiques de $\mathrm{Cu}$. Cette pratique permet d'obtenir une limite de détection de $1 \mu \mathrm{g} / \mathrm{l} \mathrm{du} \mathrm{Cu}$ dans les condensats.

Une limite de détection quasi-similaire de $2,7 \mu \mathrm{g} / \mathrm{l}$ pour l'As et de 2,5 pour Sb est trouvée par Cassella et al. (2004) en injectant $20 \mu \mathrm{l}$ d'une microémulsion constituée de $66 \%$ de naphta et de $33 \%$ d'eau acidifiée contenant du Triton $\mathrm{X}-100$. Un test effectué sur plusieurs surfactants montrent que le Triton X-100 est le plus adapté pour stabiliser les micro-émulsions.

Finalement, le Ni et le $\mathrm{V}$ sont investigués en microémulsion dans les articles de Aucelio et al. (2004) et de Vale et al. (2004). Vale et al. (2004) montre dans sa publication que le xylène est un mauvais solvant pour le sel de Ni utilisé (acide 2-ethyl hexanoique de $\mathrm{Ni}$ ) et s'oriente donc vers la microémulsion pour doser le $\mathrm{Ni}$ dans les bruts pétroliers. Un gramme de brut dilué dans $10 \mathrm{ml}$ d'eau additionné de Triton X-100 permet d'obtenir une limite de détection de $70 \mu \mathrm{g} / \mathrm{kg}$. Si la limite de détection de cet article n'est pas son point fort, deux espèces du Ni sont en revanche mis en évidence dans le brut analysé par l'intermédiaire de deux temps de pyrolyse à 400 et $1300^{\circ} \mathrm{C}$.

Le dosage du V dans les asphaltènes et dans le gasoil est entrepris par Aucelio et al. (2004) par l'intermédiaire de deux micro-émulsions différentes. Les fractions asphaltènes sont dissoutes dans le dichlorométhane avant d'être mélangé avec du propanol et de l'acide nitrique pour un mélange final contenant $4,3 \mathrm{~g} / \mathrm{l}$ d'asphaltènes. La limite de quantification du $\mathrm{V}$ dans les asphaltènes est de $4 \mu \mathrm{g} / \mathrm{g}$. Le gasoil est également mélangé en microémulsion avec du propanol et de l'acide nitrique dans deux proportions différentes de $1 / 10$ à $2 / 5$ (gasoil/volume final) et cette préparation permet une limite de quantification de $5 \mu \mathrm{g} / \mathrm{l}$ en final pour le vanadium.

\section{Applications récentes en spectrométrie d'émission à couplage inductif sur produits pétroliers}

Le dosage des produits pétroliers présente l'inconvénient d'introduire dans le plasma des quantités de solvants organiques volatiles importantes déstabilisant le plasma et par là même les conditions de mesure des métaux. De plus, les émissions provenant de la matrice organique contribuent au bruit de fond du spectre limitant la sensibilité de la technique pour la détermination des traces. On retrouve donc deux classes de publications dans la littérature récente :

- les travaux basés sur les alternatives à l'introduction directe de solvant tel que l'utilisation de nébuliseur ultrasonique pour réduire la charge en solvant, les émulsions de solvant organique en phase aqueuse, ou les minéralisations de matière organique pour les analyser en phase aqueuse (Caldiero et al., 1997) ;

- les améliorations techniques sur les appareils et applications directes.

Une des alternatives à l'introduction de solvant en quantité trop importante dans le plasma est donc l'utilisation d'un nébuliseur ultrasonique comme moyen d'introduction pour l'échantillon. L'usage d'un nébuliseur ultrasonique permet de réduire notablement la quantité de solvant injecté tout en concentrant dans le plasma l'analyte recherché. Botto (1993) utilise un nébuliseur ultrasonique (USN) pour l'analyse de produits pétroliers par ICP-OES. La fonction désolvatrice de l'USN élimine une partie importante du volume de vapeurs organiques qui arrivent dans le plasma. Le montage publié consiste en un nébuliseur ultrasonique relié à $80 \mathrm{~cm}$ de tube en verre dont la première partie est entièrement recouvert par un système chauffant en Pyrex puis refroidie par un système de refroidissement incluant une entrée et une sortie de gaz. L'échantillon organique (composés entre C6 et C8) est nébulisé à un débit d'environ $3 \mathrm{ml} / \mathrm{mn}$, l'aérosol subit une première désolvatation par évaporation dans la partie chauffée puis par condensation dans la partie réfrigérée, les vapeurs organiques non condensées étant évacuées par un drain (situé à la sortie du système refroidissant). Ce système semble pouvoir améliorer les limites de quantification d'un facteur 2 à 100 fois lors de l'injection de toluène dopé avec divers éléments. Un gain moindre est cependant observé avec les éléments $\mathrm{Al}, \mathrm{Cr}, \mathrm{Cu}, \mathrm{Pb}$ et $\mathrm{Sn}$. Cette même publication (Botto, 1993) préconise l'adjonction d'oxygène en 3 points (dans l'aérosol, en gaz externe, et dans le gaz auxiliaire) dans le plasma. Son but est de réduire l'émission de la bande moléculaire due aux composés en $\mathrm{C} 2$ dans le plasma, génante autour de $230 \mathrm{~nm}$. Dans cette application, 40 à $60 \mathrm{ml} / \mathrm{min}$ d'oxygène sont utilisé pour des vitesses d'injection de solvant organique de 1 à $4 \mathrm{ml} / \mathrm{min}$.

L'efficacité de la nébulisation du système de Botto (1993), couplé avec un condenseur ne permet pas de réduire suffisamment le volume de gaz envoyé dans le plasma lorsqu'il s'agit de solvant très volatile (coupes ou produits ayant un point d'ébullition inférieur à $100^{\circ} \mathrm{C}$ ). Botto et Zhu (1994) recommandent alors d'y ajouter une membrane de désolvatation de $80 \mathrm{~cm}$ de long qui permet encore de réduire la quantité finale de solvant dans le plasma. Le principe est basé sur l'adjonction, suite au nébuliseur ultrasonique, d'une membrane dans laquelle circulent dans un sens le mélange de gaz et aérosols envoyés vers le plasma alors que de l'argon sec circule à contre courant avec un débit de $0,7 \mathrm{ml} / \mathrm{min}$. Au cours du passage de l'aérosol dans la membrane de désolvatation, les vapeurs organiques résiduelles sont diffusées à travers des pores de $2 \mu \mathrm{m}$ de diamètre dans les parois de la membrane. Le contre courant d'argon sec emporte les vapeurs organiques à l'extérieur de l'unité, permettant une 
désolvatation de $99 \%$ du volume des vapeurs organiques entrant (Botto et Zhu, 1994).

Une amélioration d'un facteur 2 à 10 fois est observé sur les limites de détection pour les éléments tel que $\mathrm{Ag}, \mathrm{Al}, \mathrm{Cd}$, $\mathrm{Cr}, \mathrm{Cu}, \mathrm{Mg}, \mathrm{Mo}, \mathrm{Na}, \mathrm{Ni}, \mathrm{Pb}$ et $\mathrm{Sn}$ alors qu'aucun progrès n'est enregistré pour le $\mathrm{Ba}, \mathrm{Ca}, \mathrm{Fe}, \mathrm{Mn}, \mathrm{P}, \mathrm{Ti}$, V et $\mathrm{Zn}$ (voir tableau 1) vis-à-vis de la publication de Botto (1993). L'usage de l'oxygène en gaz d'appoint permet une amélioration notable de la détection des métaux en ICP-OES dans cette application.

Un des soucis majeurs de la désolvatation par membrane utilisée avec les produits pétroliers est de savoir ce qui passe au travers de la membrane et ce qui y reste. L'usage de cette méthode, dans le domaine de la pétrochimie avec des solvants composés d'un seul type de molécule (éthanol, acétone, dichlorométhane ou toluène), est aisé puisque seul le solvant peut être éliminé. Dans le cas de produits pétroliers, composés de plusieurs centaines de produits organiques différents, il est très difficile de savoir quels sont les composés éliminés et ceux retenus. Il en est donc de même avec les composés organométalliques qui peuvent être éliminés par la membrane avant dosage et fausser donc la mesure. Dans Botto et Zhu (1994), le Si et le B ne sont pas dosés dans la solution de type Spex, car présents sous forme volatiles et donc éliminés par le système de désolvatation. De même le dosage du tétra-éthyl de plomb n'est possible qu'a près l'avoir piégé sous forme de $\mathrm{PbBr}_{2}$, espèce non-volatile. Des problèmes d'encrassage de la membrane sont également observés, rendant cette application impossible pour l'analyse d'huile brute ou de fractions lourdes. Cette application reste cependant utilisable pour la mesure de métaux dans les substances organiques composés d'une seule molécule tel que cela peut être le cas en pétrochimie.
Dans Botto et Zhu (1996), l’USN équipé de la membrane de désolvatation est encore modifié dans le but de pouvoir doser les composés de C4 à C8 sans influence majeure du solvant utilisé. Un allongement de la membrane passant de $80 \mathrm{~cm}$ à $140 \mathrm{~cm}$ permet d'améliorer encore l'élimination des fractions légères avec un rendement de désolvatation allant jusqu'à 99,9\%. Le but avoué de cette publication est de rendre possible une calibration universelle quelque soit la matrice organique injectée entre $\mathrm{C} 4$ et $\mathrm{C} 8$. Les limites de détection sont meilleures que le système précédent (Botto et $\mathrm{Zhu}, 1994)$ pour les éléments $\mathrm{Cr}, \mathrm{Fe}, \mathrm{Mn}, \mathrm{Na}, \mathrm{Ni}, \mathrm{Pb}$ et $\mathrm{V}$, elles sont en revanche dégradées pour l' $\mathrm{Ag}, \mathrm{Cu}, \mathrm{Mg}$ et $\mathrm{Sn}$, et comparables pour l'Al, Ca, Cd, Mo et Zn (voir tableau 1).

L'injection d'oxygène dans le plasma semble une solution retenue par plusieurs auteurs travaillant en ICP-OES, bien que cette pratique soit plus communément associée à l'injection de solvants carbonés en ICP-MS. Edlund et al. (2002) ajoute 350 1/min d'oxygène dans le gaz de nébulisation $(800 \mathrm{ml} / \mathrm{min})$ après avoir testé des rajouts d'oxygène dans le gaz nébuliseur, l'intermédiaire et le gaz externe. Cet ajout a pour effet d'améliorer par un facteur 50 le dosage du Na et de 30 pour le potassium dans les biodiesels. Des limites de détection de 2 et $7 \mathrm{ppb}$ pour $\mathrm{Na}$ et $\mathrm{K}$ respectivement sont atteintes, alors qu'aucun effet n'est observé sur le spectre du $\mathrm{Ca}, \mathrm{Mg}, \mathrm{P}$ et $\mathrm{Cl}$.

L'utilisation de l'oxygène est également retenu par Krengel-Rothensee et al. (1999) dans son dosage du Cl, Br, I, $\mathrm{S}$ et $\mathrm{P}$ dans l'huile avec le développement d'une méthode basée sur les raies observables dans l'UV (130-190 nm). $80 \mathrm{ml} / \mathrm{min}$ sont ajoutés au gaz auxiliaire afin d'éviter les dépôts de carbone sur l'injecteur en quartz et minimiser les interférences spectrales dues au carbone. Les limites de quantification obtenues sont reportées dans le tableau 2.

TABLEAU 1

Comparaisons des limites de quantification ds Botto (1993) et Botto et Zhu (1996)

Quantification limit obtained by Botto (1993) and Botto and Zhu (1996)

\begin{tabular}{|c|c|c|c|c|c|c|c|c|c|c|}
\hline Éléments & $\mathrm{Ag}$ & $\mathrm{Al}$ & As & $\mathrm{Ba}$ & $\mathrm{Ca}$ & $\mathrm{Cd}$ & $\mathrm{Cr}$ & $\mathrm{Cu}$ & $\mathrm{Fe}$ & $\mathrm{Mg}$ \\
\hline $\begin{array}{l}\text { LOD ds pentane }(\mu \mathrm{g} / \mathrm{l}) \text {, } \\
\text { Botto et Zhu (1996) }\end{array}$ & 3 & 2 & 20 & 0,04 & 1 & 0,3 & 0,4 & 0,8 & 0,2 & 0,3 \\
\hline $\begin{array}{l}\text { LOD ds toluène }(\mu \mathrm{g} / \mathrm{kg}) \text {, } \\
\text { Botto et Zhu (1994) }\end{array}$ & 0,7 & 1,5 & - & 0,2 & 1 & 0,2 & 1,3 & 0,1 & 2 & 0,1 \\
\hline $\begin{array}{l}\text { LOD ds toluène }(\mu \mathrm{g} / \mathrm{kg}) \text {, } \\
\text { Botto (1993) }\end{array}$ & 1,8 & 4,9 & - & 0,18 & 1,9 & 0,81 & 14 & 0,74 & 1,6 & 1,1 \\
\hline Éléments & $\mathrm{Mn}$ & Mo & $\mathrm{Na}$ & $\mathrm{Ni}$ & $\mathrm{Pb}$ & $\mathrm{Si}$ & $\mathrm{Sn}$ & V & $\mathrm{Zn}$ & \\
\hline $\begin{array}{l}\text { LOD ds pentane }(\mu \mathrm{g} / \mathrm{l}) \text {, } \\
\text { Botto et Zhu (1996) }\end{array}$ & 0,05 & 1 & 0,5 & 0,2 & 0,8 & 0,6 & 25 & 0,06 & 0,2 & \\
\hline $\begin{array}{l}\text { LOD ds toluène }(\mu \mathrm{g} / \mathrm{kg}) \text {, } \\
\text { Botto et Zhu (1994) }\end{array}$ & 0,2 & 2 & 1,3 & - & - & - & - & 1 & 0,3 & \\
\hline $\begin{array}{l}\text { LOD ds toluène }(\mu \mathrm{g} / \mathrm{kg}) \text {, } \\
\text { Botto (1993) }\end{array}$ & 0,39 & 7,8 & 2,9 & 11 & 11 & 2,7 & 47 & 1,9 & 1,1 & \\
\hline
\end{tabular}


TABLEAU 2

Limites de détection dans les huiles et biodiesel par ICP en torche organique

Detection limit in lubricant and biodiesel with organic injection in ICP

\begin{tabular}{l|c|c|c|c|c|c|c|c|c}
\hline Éléments & $\begin{array}{c}\mathrm{Cl} \\
134,724 \\
\mathrm{~nm}\end{array}$ & $\begin{array}{c}\mathrm{Br} \\
164,34 \\
\mathrm{~nm}\end{array}$ & $\begin{array}{c}161,76 \\
\mathrm{~nm}\end{array}$ & $\begin{array}{c}\mathrm{P} \\
177,50 \\
\mathrm{~nm}\end{array}$ & $\begin{array}{c}\mathrm{S} \\
180,70 \\
\mathrm{~nm}\end{array}$ & $\begin{array}{c}\mathrm{Na} \\
589,592 \\
\mathrm{~nm}\end{array}$ & $\begin{array}{c}\mathrm{K} \\
766,490 \\
\mathrm{~nm}\end{array}$ & $\begin{array}{c}\mathrm{Mg} \\
279,553 \\
\mathrm{~nm}\end{array}$ & $\begin{array}{c}\mathrm{Ca} \\
393,366 \\
\mathrm{~nm}\end{array}$ \\
\hline $\begin{array}{l}\text { LOD dans biodiesel (ppm) } \\
\text { (Edlund } \text { et al., 2002) }\end{array}$ & 0,4 & $\mathrm{ND}$ & $\mathrm{ND}$ & 0,032 & $\mathrm{ND}$ & 0,002 & 0,007 & 0,001 & 0,0004 \\
\hline $\begin{array}{l}\text { LOD dans huiles (ppm) } \\
\text { (Krengel-Rothense } \text { et al., 1999) }\end{array}$ & 0,9 & 1,6 & 0,47 & 0,04 & 0,07 & $\mathrm{ND}$ & $\mathrm{ND}$ & $\mathrm{ND}$ & $\mathrm{ND}$ \\
\hline
\end{tabular}

Une autre alternative pour limiter l'introduction de matières organiques dans le plasma consiste à injecter le solvant organique dispersé dans une phase aqueuse. Wang et al. (2003) ont déterminés 22 métaux en teneurs de l'ordre de la dizaine de $\mathrm{mg} / \mathrm{kg}$ dans un extrait organique pharmaceutique, une huile de vidange et un produit de référence NIST, en dispersant $50 \mu \mathrm{l}$ d'échantillon dans $20 \mathrm{ml}$ d'acide nitrique concentré. L'ICP fonctionne alors en mode aqueux, les limites de quantification vont de $0,1 \mathrm{à} 2 \mathrm{mg} / \mathrm{kg}$ dans les matrices organiques du fait de la forte dilution de l'échantillon dans l'acide. De la même façon, Druon et al. (2000) mélange $1,5 \mathrm{~g}$ de bitume à $100 \mathrm{ml}$ d'eau avec $5 \mathrm{ml}$ de xylène et $2 \mathrm{ml}$ de Triton 100. Les limites de détection sur le Ni et le $\mathrm{V}$ sont de $0,4 \mathrm{mg} / \mathrm{kg}$ et $1,2 \mathrm{mg} / \mathrm{kg}$ respectivement.

Un travail de comparaison de trois modes d'introduction d'huile lubrifiante usagée a été entrepris par Souza et al. (2004). Ainsi ont été comparés :

- l'introduction en mode aqueux après minéralisation acide de $200 \mathrm{mg}$ en bombe $\mu$-onde fermée, avec des dilutions finales de 50 fois ;

- la dilution directe dans le kérozène avec un facteur de dilution de 20 ;

- le dosage en mode aqueux après mise en émulsion de $0,04 \mathrm{~g}$ d'échantillon dans $10 \mathrm{~g}$ d'eau.

Les limites de quantification, données dans le tableau 3, montrent que les limites de quantification en mode aqueux après minéralisation sont meilleures, à cause des interférences de la matière organique qui apportent une fluctuation plus importante du signal avec les deux autres types de dosage.

TABLEAU 3

Limites de quantification en $\mathrm{mg} / \mathrm{kg}$ dans Souza et al. (2004)

Quantification limit ( $\mathrm{mg} / \mathrm{kg}$ ) in Souza et al. (2004)

\begin{tabular}{l|c|c|c|c|c}
\hline Éléments & $\mathrm{Ni}$ & $\mathrm{Mo}$ & $\mathrm{Cr}$ & $\mathrm{V}$ & $\mathrm{Ti}$ \\
\hline Émulsion & 0,7 & 1,7 & 0,9 & 0,6 & 0,3 \\
\hline Minéralisation $\mu$-onde & 0,5 & 0,9 & 0,06 & 0,4 & 0,2 \\
\hline Dilution organique & 6 & 5,1 & 0,9 & 3,4 & 0,7 \\
\hline
\end{tabular}

Dans le cas de ce travail, un faible ajout d'oxygène dans le plasma $(0,0488 \mathrm{ml} / \mathrm{min})$ est également fait et semble améliorer la sensibilité de la mesure, malgré la faible quantité de matières organiques injectées par l'émulsion (6\% d'organique dans un débit de $0,8 \mathrm{ml} / \mathrm{min}$, soit $0,048 \mathrm{ml} / \mathrm{min}$ ). Des observations faites également sur différents modes d'injection montrent que l'utilisation d'un nébuliseur de type Meinhard associé à une chambre de nébulisation cyclonique est optimal alors que l'usage d'un nébuliseur de type $\mu$-mist provoque des bouchages. Finalement, l'utilisation d'un nébuliseur de type flux croisés associé à une chambre de nébulisation de Scott donne une sensibilité moindre. L'usage d'étalons aqueux dans les émulsions pour les calibrations fonctionne avec l'injection Meinhardt + chambre cyclonique, mais ce n'est plus le cas avec les autres modes d'injection.

\section{Applications récentes en spectrométrie de masse par plasma à couplage inductif sur produits pétroliers}

La problématique de l'introduction de solvant organique en quantité trop importante dans le plasma est d'autant plus importante en ICP-MS pour deux raisons :

- comme en ICP optique, le plasma est instable par la charge de produits volatiles reçue;

- le carbone forme des ions polyatomiques avec l'argon et les métaux, qui sont gênants pour la détermination de nombreux éléments et provoque l'encrassement des cônes.

On retrouve donc dans la littérature sur ce sujet toutes les approches permettant de limiter le flux de carbone au sein du plasma :

- l'utilisation de nébuliseur ultrasonique comme moyen d'introduction pour l'échantillon;

- l'introduction de l'échantillon de façon séquentielle ou par vaporisation électrothermique ;

- l'utilisation de micro-nébuliseur réduisant le flux de solvant dans le plasma;

- la formation de micro-émulsion permettant de travailler en mode aqueux ; 
- la minéralisation de l'échantillon organique avant introduction du minéralisat en mode aqueux.

L'usage d'un nébuliseur ultrasonique, éventuellement couplé à une membrane de désolvatation, permet de réduire notablement la quantité de solvant injecté tout en concentrant dans le plasma l'analyte recherché. Ainsi plusieurs auteurs (Duyck et al., 2002 ; Brenner et al., 1997 ; Olsen et al., 1997 ; Alves et al., 1994) se sont tournés vers cette solution pour différentes applications.

Beaucoup d'auteurs ont cependant travaillé sur des solvants légers ou des coupes légères. Ainsi Alves et al. (1994) utilise un nébuliseur ultrasonique couplé avec un système permettant un premier passage dans la glace carbonique $\left(-77^{\circ}\right)$, suivi de 4 boucles passant dans un bain à $-80^{\circ} \mathrm{C}$ puis réchauffées à une température de $100^{\circ} \mathrm{C}$ avant injection dans l'ICP-MS. Le système est testé pour analyser les métaux traces dans l'éthanol, le méthanol, l'acétone et l'acétonitrile et les interférences avec les espèces $\mathrm{CO}^{+}$(pour la masse $\left.{ }^{28} \mathrm{Si}^{+}\right), \mathrm{CO}_{2}^{+}, \mathrm{ArC}^{+}\left({ }^{52} \mathrm{Cr}^{+}\right)$et $\mathrm{ArO}^{+}\left({ }^{56} \mathrm{Fe}^{+}\right)$sont particulièrement étudiées. Cette procédure semble plutôt performante pour désolvater l'effluent puisque l'ajout d'oxygène ( $80 \mathrm{ml} / \mathrm{min}$ ) n'est nécessaire de façon permanente que pour l'injection d'éthanol (injection liquide à $1,5 \mathrm{ml} / \mathrm{min}$ ). Dans le cas de l'acétone, l'injection d'oxygène pendant seulement 30 secondes sur 2 heures de mesure permet de nettoyer le cône d'échantillonage et de revenir au signal initial.

Des essais, effectués avec différentes espèces métalliques du $\mathrm{Co}, \mathrm{Nb}$ et de l'Y ayant des points d'ébullition similaires à ceux des solvants éliminés, ne montrent pas une perte significative sur ces métaux, contrairement aux travaux de Botto et Zhu (1994) et Olsen et al. (1997) effectués sur un nébuliseur ultrasonique couplé avec une membrane de désolvatation.

En revanche, un effet mémoire important est mis en évidence lors de l'utilisation de sels de $\mathrm{Nb}$ organiques volatiles (Alves et al., 1994) ou des composés du mercure (Olsen et al., 1997), obligeant des temps de rinçage très longs.

Dans l'étude de Brenner et al. (1997), le solvant utilisé est le chloroforme et 14 éléments $(\mathrm{V}, \mathrm{Cr}, \mathrm{Mn}, \mathrm{Ni}, \mathrm{Cu}, \mathrm{Fe}, \mathrm{Mo}$,
$\mathrm{Ag}, \mathrm{Zn}, \mathrm{Sn}, \mathrm{Tl}, \mathrm{Cd}, \mathrm{Bi}$ et $\mathrm{Pb}$ ) sont mesurés dans des extraits au solvant. L'ajout d'oxygène est dans ce travail nécessaire (30 $\mathrm{ml} / \mathrm{min}$ ) pour limiter l'interférence des polyatomiques et minimiser les dépôts de carbone sur les cônes d'échantillonage. Dans le cas de ce travail, l'utilisation du nébuliseur ultrasonique équipé d'une membrane de désolvatation de $1,5 \mathrm{~m}$ est extrêmement important pour retirer le chloroforme qui provoque une interférence polyatomique majeure sur le $\mathrm{V}$ $\left({ }^{35} \mathrm{Cl}^{16} \mathrm{O}^{+}\right.$pour $\left.{ }^{51} \mathrm{~V}^{+}\right)$.

Finalement Duyck et al. (2002) utilise aussi un nébuliseur ultrasonique pour le dosage de 19 métaux dans les huiles brutes du bassin de Potiguar. Pour limiter le dépôt des fractions asphalténiques dans le tube du nébuliseur, le chauffage de ce dernier est déconnecté.

Les limites de détection de Brenner et al. (1997), Olsen et al. (1997) et Duyck et al. (2002), ayant travaillé avec un nébuliseur ultrasonique sont reportées dans le tableau 4 .

Les limitations de ce système reste la perte partielle ou totale d'élément volatile, et des discriminations entre différents composés. De plus, l'utilisation de membrane de désolvatation n'est pas applicable aux fractions moyennes et lourdes du pétrole pour cause d'encrassement de la membrane.

Un autre moyen de réduire l'apport de carbone dans le plasma réside dans l'introduction séquentielle de l'échantillon permettant de limiter le flux continu de carbone. C'est la vaporisation électrothermique (ETV) où la matrice est détruite par une montée en température dans un four de type graphite avant envoi dans le plasma (Resano et al. (2001), Escobar et al. (1996), Saint'Pierre et al., (2002)). Le premier avantage de cette approche est de pouvoir séparer l'analyte d'intérêt de la matrice organique par un programme en température adéquat. Ceci permet d'éliminer les interférences liées à la matrice et celles liées à l'ajout d'oxygène, devenu inutile. Un autre avantage réside dans le volume consommé, puisqu'une dizaine de $\mu \mathrm{l}$ sont injectés dans le four. Cette technique s'avère donc très utile lorsqu'une quantité limitée de produit est disponible. En revanche, on retrouve avec ce

TABLEAU 4

Limites de détection par nébulisation ultrasonique (USN) et ICP-MS

Detection limit with ultrasonic nebulizer (USN) and ICP-MS

\begin{tabular}{l|c|c|c|c|c|c|c|c|c|c|c|c|c}
\hline Éléments & ${ }^{51} \mathrm{~V}$ & ${ }^{52} \mathrm{Cr}$ & ${ }^{55} \mathrm{Mn}$ & $\begin{array}{c}{ }^{56} \mathrm{Fe} / \\
{ }^{57} \mathrm{Fe}\end{array}$ & $\begin{array}{c}{ }^{58} \mathrm{Ni} / \\
{ }^{60} \mathrm{Ni}\end{array}$ & $\begin{array}{c}{ }^{6} \mathrm{Cu} / \\
{ }^{62} \mathrm{Cu}\end{array}$ & ${ }^{66} \mathrm{Zn}$ & $\begin{array}{c}{ }^{95} \mathrm{Mo} / \\
{ }^{98} \mathrm{Mo}\end{array}$ & $\begin{array}{c}{ }^{107} \mathrm{Ag} / \\
{ }^{109} \mathrm{Ag}\end{array}$ & ${ }^{111} \mathrm{Cd}$ & ${ }^{118} \mathrm{Sn}$ & ${ }^{208} \mathrm{~Pb}$ & ${ }^{209} \mathrm{Bi}$ \\
\hline $\begin{array}{l}\text { Brenner } \text { et al., } 1997 \\
\left(\mathrm{USN}-\mathrm{CHCl}_{3}, \mu \mathrm{g} / \mathrm{kg}\right)\end{array}$ & 0,75 & 0,17 & 0,011 & $\begin{array}{c}0,12 / \\
0,20\end{array}$ & $\begin{array}{c}0,042 / \\
0,015\end{array}$ & $\begin{array}{c}0,20 / \\
0,22\end{array}$ & 0,30 & $\begin{array}{c}0,012 / \\
0,007\end{array}$ & $\begin{array}{c}0,011 / \\
0,018\end{array}$ & 0,015 & 0,16 & 0,002 & 0,003 \\
\hline $\begin{array}{l}\text { Olsen } \text { et al. }, 1997 \\
\text { (USN-xylène, } \mu \mathrm{g} / \mathrm{kg})\end{array}$ & 0,009 & $\mathrm{ND}$ & $\mathrm{ND}$ & $\mathrm{ND}$ & 0,006 & $\mathrm{ND}$ & $\mathrm{ND}$ & $\mathrm{ND}$ & $\mathrm{ND}$ & 0,004 & $\mathrm{ND}$ & $\mathrm{ND}$ & 0,004 \\
\hline $\begin{array}{l}\text { Duyck et } \text { al. }, 2002 \\
\text { (USN-toluène, } \mu \mathrm{g} / \mathrm{l})\end{array}$ & 0,02 & $\mathrm{ND}$ & 0,6 & $\begin{array}{c}-/ \\
0,5\end{array}$ & $\begin{array}{c}-/ \\
0,1\end{array}$ & $\begin{array}{c}0,1 / \\
0,1\end{array}$ & 0,35 & 0,04 & $\begin{array}{c}0,15 / \\
0,25\end{array}$ & 0,1 & 0,15 & 0,2 & $\mathrm{ND}$ \\
\hline
\end{tabular}


mode d'introduction les inconvénients de l'introduction par four graphite :

- l'ajout éventuel de modificateurs est nécessaire selon les métaux à doser ;

- l'aspect mono-élémentaire de la technique.

Ainsi, Escobar et al. (1996) ont développé cette méthode pour doser l' $\mathrm{Al}, \mathrm{Fe}, \mathrm{Mg}$ et l'Y dans les huiles lubrifiantes, alors que Saint'Pierre et al. (2002) ont analysés le $\mathrm{Cu}, \mathrm{Mn}$, $\mathrm{Ni}$ et $\mathrm{Sn}$ dans les essences. La capacité à mesurer plusieurs éléments sur le signal transitoire émis par l'ETV a été étudiée par Resano et al. (2001). Les éléments étant injectés dans le flux d'argon uniquement pendant la période de vaporisation, qui dure environs 10 secondes, la mesure de ceux-ci dans le mode ETV doit s'effectuer en ICP-MS dans cet intervalle de temps court. L'ICP-MS quadrupolaire étant séquentiel, la mesure de plusieurs isotopes requiert un balayage du ratio $\mathrm{m} / \mathrm{Z}$. Ainsi, plus le nombre d'isotopes mesurés est important, plus le cycle de balayage du quadrupole est long, passant de 0,15 seconde pour 12 isotopes à 1,5 seconde pour 96 isotopes. Le nombre de point de mesure sur le signal transitoire diminue ainsi avec le nombre d'isotopes mesurés. Une dégradation franche de la limite de détection est observée à partir de 24 isotopes mesurés. Ce travail ne prend en revanche pas en compte l'usage de rampe de température et de modificateurs qui sont souvent choisis en fonction d'un élément et limitent par conséquent les développements multi-élémentaires. Les limites de quantifications obtenues sur les produits pétroliers (voir tableau 5) sont identiques à celles obtenues avec les autres techniques d'introduction en ICP-MS. En revanche, la quantité d'échantillons injectée se limite à quelques dizaines de $\mu \mathrm{l}$.

L'utilisation de micro-nébuliseur réduisant le flux de solvant dans le plasma est une troisième alternative retenue par de nombreux auteurs. Plusieurs micro-nébuliseurs existent sur le marché fonctionnant de diverses façons. Ils nécessitent une chambre de nébulisation (cas des nébuliseurs de type $\mathrm{PFA}^{\circledR}$ ) avec une partie de l'échantillon évacuée vers le drain, ou permettent l'injection directe dans le plasma (cas du DIN : nébuliseur à injection directe et DIHEN : nébuliseur à injection directe à haute efficacité) avec $100 \%$ de l'échantillon transféré vers la torche.

Botto (2002) a utilisé la nébulisation par injection directe (DIN) pour l'analyse de naphtas. Le DIN est un nébuliseur à faible débit $(60 \mu \mathrm{l} / \mathrm{min})$ directement connecté à la torche commercialisé par CETAC. Grâce à son faible débit, le DIN permet de mesurer des échantillons pétroliers en introduisant dans le plasma de l'ICP-MS une quantité limitée de vapeurs organiques. Les échantillons de naphtas sont dilués 5 fois dans le xylène, les distillats moyens entre 10 à 50 fois et les huiles lourdes 50 fois. Des étalons internes contenant du ${ }^{59} \mathrm{Co},{ }^{89} \mathrm{Y}$ et ${ }^{209} \mathrm{Bi}$ sont ajoutés à l'échantillon pour pouvoir compenser les dérives instrumentales rencontrées lors de l'analyse avec le DIN. Les variations sur les produits légers analysés sont inférieures à $20 \%$ et jugées comme acceptables pour l'analyse de charge. Kahen et al. (2002) a utilisé un nébuliseur à injection directe à haute efficacité (DIHEN). Ce nébuliseur (débit $=10 \mu 1 / \mathrm{min}$ ) est le modèle qui a remplacé le DIN sur le marché. La position du DIHEN dans la torche à plasma est cruciale pour le rendement du signal observé, une variation de $5 \mathrm{~mm}$ occasionnant des diminutions de réponse d'un facteur 2 à 3 .

Dans le cas des deux injecteurs mentionnés (DIN et DIHEN), l'échantillon est introduit dans le plasma par une boucle de $1000 \mu$ l pour Botto (2002) et de $20 \mu$ l pour Kahen et al. (2003), à l'aide d'une vanne 6-voies. Le restant du temps, du xylène est passé en direct dans le plasma via le nébuliseur. Cette configuration a pour but de limiter le volume d'échantillon passé dans le nébuliseur, limitant

TABLEAU 5

Limites de détection en ICP-MS avec différents types d'injections

Detection limit with different injection device in ICP-MS

\begin{tabular}{|c|c|c|c|c|c|c|c|c|c|c|c|}
\hline Éléments & ${ }^{51} \mathrm{~V}$ & ${ }^{55} \mathrm{Mn}$ & $\begin{array}{l}{ }^{56} \mathrm{Fe} / \\
{ }^{57} \mathrm{Fe}\end{array}$ & $\begin{array}{l}{ }^{58} \mathrm{Ni} / \\
{ }^{60} \mathrm{Ni}\end{array}$ & $\begin{array}{l}{ }^{63} \mathrm{Cu} / \\
{ }^{62} \mathrm{Cu}\end{array}$ & ${ }^{66} \mathrm{Zn}$ & $\begin{array}{l}{ }^{95} \mathrm{Mo} / \\
{ }^{98} \mathrm{Mo}\end{array}$ & $\begin{array}{l}{ }^{107} \mathrm{Ag} / \\
{ }^{109} \mathrm{Ag}\end{array}$ & ${ }^{111} \mathrm{Cd}$ & ${ }^{118} \mathrm{Sn}$ & ${ }^{208} \mathrm{~Pb}$ \\
\hline $\begin{array}{l}\text { Kahen } \text { et al., } 2003 \text { - DIHEN } \\
\text { (xylène, } \mu \mathrm{g} / \mathrm{kg} \text { ) }\end{array}$ & 0,87 & ND & $\begin{array}{c}-/ \\
0,81\end{array}$ & ND & ND & ND & ND & ND & 0,77 & ND & 1,46 \\
\hline $\begin{array}{l}\text { Botto }(2002) \text { - DIN } \\
\text { (hexane, } \mu \mathrm{g} / \mathrm{kg} \text { ) }\end{array}$ & 0,08 & 0,04 & ND & $\begin{array}{l}-1 \\
0,2\end{array}$ & $\begin{array}{c}0,07 / \\
-\end{array}$ & 0,03 & $\begin{array}{c}0,2 / \\
-\end{array}$ & ND & 0,08 & 0,03 & 0,1 \\
\hline $\begin{array}{l}\text { Botto }(2002) \text { - DIN } \\
\text { (xylène, } \mu \mathrm{g} / \mathrm{kg} \text { ) }\end{array}$ & 3.8 & 0,5 & $\begin{array}{l}-1 \\
5\end{array}$ & $\begin{array}{l}-1 \\
2\end{array}$ & $\begin{array}{c}0,2 / \\
-\end{array}$ & 0,6 & $\begin{array}{c}0,1 / \\
-\end{array}$ & ND & 2 & 0,06 & 0,3 \\
\hline $\begin{array}{l}\text { Kumar et Gangadharan (1999) - } \\
\mu \text {-émulsion, } \mu \mathrm{g} / \mathrm{kg}\end{array}$ & 0,6 & ND & ND & 0,7 & ND & ND & ND & ND & ND & ND & 0,09 \\
\hline $\begin{array}{l}\text { Saint'Pierre et al. (2002) - ETV } \\
\text { essence, } \mu \mathrm{g} / 1\end{array}$ & ND & 0,02 & ND & $\begin{array}{c}-1 \\
0,38\end{array}$ & 0,22 & ND & ND & ND & ND & 0,03 & ND \\
\hline
\end{tabular}


d'autant l'encrassement de l'injecteur. Elle permet aussi d'éviter la déstabilisation du plasma par commutation entre échantillon et solvant de rinçage, provoquant le passage d'une bulle d'air dans l'injecteur. De l'oxygène est également ajouté aux deux systèmes d'introduction $(0-300 \mathrm{ml} / \mathrm{min}$ pour Botto, 2002 et $30 \mathrm{ml} / \mathrm{min}$ pour Kahlen et al., 2003) afin de limiter le bouchage des $\mu$-nébuliseurs et limiter le dépôt de carbone sur les cônes de prélèvement. Les performances des deux méthodes (DIN et DIHEN) sont comparables (limites de détections reportées dans le tableau 5) bien que les bouchages soient visiblement plus fréquents avec le DIHEN. L'avantage principal de ces deux $\mu$-nébuliseurs réside essentiellement dans le fait que les éléments volatiles n'ont aucune opportunité de s'échapper avant leur entrée dans le plasma, répondant au principal désavantage de la nébulisation ultrasonique.

Dreyfus et al. (2005) ont mis au point une méthode permettant le dosage des métaux traces dans les huiles brutes et ses fractions (maltènes et asphaltènes) par injection avec un micro-nébuliseur de type PFA-100 associé à l'utilisation d'une chambre de Scott refroidie. Un débit d'oxygène de $66 \mathrm{ml} / \mathrm{min}$ permet de limiter le dépôt de carbone sur les cônes de platine et d'assurer une combustion complète de l'échantillon. Les dosages sont effectués par ajouts dosés pour limiter les effets de matrices et interférences associées. Les limites de détection atteintes sont situées entre 0,01 et $0,2 \mu \mathrm{g} / \mathrm{kg}$. Un système de filtration en téflon permet la séparation des fractions pétrolières par précipitation des asphaltènes dans le $\mathrm{n}$-heptane. Après redilution adéquate dans le xylène, les fractions sont introduites dans le plasma et les mesures de $\mathrm{Ni}, \mathrm{V}$, $\mathrm{Cu}, \mathrm{Mo}, \mathrm{Ag}, \mathrm{Sn}, \mathrm{Ba}$, et $\mathrm{Pb}$ montrent un bilan de masse proche de $100 \%$. Les résultats montrent que les éléments analysés sont hautement concentrés dans les fractions asphalténiques et que l'utilisation d'un injecteur de plus faible diamètre $(1,14 \mathrm{~mm}$ contre $1,5 \mathrm{~mm})$ permet une amélioration des limites de détection d'un facteur deux au minimum.

Al-Swaidan et Al-Gadi (2002), et Al-Swaidan (1996) ont préférés fonctionner en mode aqueux et une des deux alternatives pour maintenir l'ICP en injection aqueuse consiste à transformer l'échantillon organique en une micro-émulsion permettant de travailler en mode aqueux. Le ratio choisit entre phase aqueuse et phase organique est de 100 , avec $0,5 \mathrm{~g}$ d'échantillon dilué avec un mélange de Triton $(0,5 \mathrm{ml}), 5 \mathrm{ml}$ d'acide nitrique à $40 \%, 1,5 \mathrm{ml}$ de tétraline comme solvant pour l'échantillon organique et le tout ajusté à $50 \mathrm{ml}$ avec de l'eau. La micro-émulsion est introduite séquentiellement dans le plasma à l'aide d'une boucle de $60 \mu 1$ de façon à réduire encore l'impact des composés organiques sur la stabilité du plasma. Il n'est donc pas nécessaire d'introduire de l'oxygène dans le plasma pour limiter le dépôt de carbone sur les cônes d'échantillonnage. Le $\mathrm{Pb}, \mathrm{Ni}$ et le $\mathrm{V}$ ont été dosés par cette technique dans trois bruts, des gazoles, des kérozènes et des essences. Aucune limite de détection n'est donnée dans ces articles puisque les trois éléments recherchés sont en concentrations suffisantes. Malgré une dilution de cent fois pour l'essence, le kérosène et le gazole dans la micro-émulsion, les pentes de la calibration sont différentes entre les 3 matrices injectées et nécessite donc un dosage par ajouts dosés.

Dans le domaine des émulsions, Kumar et Gangadharan (1999) adoptent une expérience différente en effectuant une émulsion plus riche en phase organique qu'en phase aqueuse. Cette approche de l'émulsion est unique dans la littérature à ce jour et donne au regard des limites de détection annoncées (voir tableau 5) des résultats intéressants. Le ratio choisit entre phase aqueuse et phase organique est de 0,5 , avec $2 \mathrm{ml}$ d'échantillon dilué avec un $\mathrm{ml}$ de Triton 2,5\%. La calibration est effectuée en remplaçant l'échantillon par des étalons de xylène, le remplacement du xylène par des solutions aqueuses dans l'émulsion pour la calibration faussant la justesse de la mesure. Ce mélange, selon les auteurs, réduit fortement l'interférence de $\mathrm{ArC}^{+}$et permet des limites de détection de $0,05 \mathrm{ppb}$ pour le Co, 0,1 ppb pour l'As et $0,12 \mathrm{ppb}$ pour le $\mathrm{Hg}$, non données dans le tableau 5 .

La dernière alternative que l'on trouve dans la littérature, permettant de conserver l'ICP en mode aqueux et limitant l'introduction de carbone dans le plasma, consiste en la minéralisation de l'échantillon organique avant introduction du minéralisat en mode aqueux. Cette procédure, utilisée par Bing-Quan et al. (2001) sur des kérogènes, huiles brutes et bitumes, nécessite de détruire la matrice organique. La destruction en système ouvert a le désavantage de ne pas être quantitatif pour certains éléments pouvant être perdus au cours de la minéralisation. Lachas et al. (2000) compare les résultats obtenus sur une huile certifiée après une minéralisation par voie humide à l'acide sulfurique et une extraction par micro-onde sur des quantités de matière de $20 \mathrm{mg}$. Bien que l'extraction ne permette pas de s'assurer que la totalité des métaux présents soit disponible, les résultats obtenus par cette voie sont meilleurs que ceux obtenus en mode humide, surtout pour les éléments $\mathrm{Cr}, \mathrm{Cu}$, Mo et $\mathrm{Sn}$ sous-dosés en mode humide et donc partiellement perdus lors de la minéralisation. Une dernière alternative pour passer les éléments d'intérêt en phase aqueuse consiste en la minéralisation en bombe de combustion et récupération des produits de combustion dans une solution basique. Cette technique de préparation est issue des méthodes dosant les halogènes et est justement utilisée par Begak et Syroezhko (2004) pour doser les halogènes $\mathrm{Cl}, \mathrm{Br}$ et I organiques dans les produits de type essence et kérosène entre 0,5 et $20 \mathrm{mg} / \mathrm{l}$ par ICP-MS.

Parmi les autres façons de minéraliser les produits pétroliers, la digestion par micro-onde en bombe fermée est une alternative de plus en plus massivement retenue par la communauté. Ce protocole permet de monter en pression et en température tout en maintenant dans un espace clos les espèces volatiles habituellement perdues par les voies de minéralisations plus classiques. En revanche, le fait que le système soit clos limite la prise d'essai de l'échantillon. Ce 
dernier provoque en effet, par sa dégradation, de grandes quantités de gaz, faisant rapidement monter la pression dans l'enceinte de la réaction. Cette limitation peut s'avérer être un avantage lorsque le produit à analyser n'est disponible qu'en très faible quantité. Ainsi, Lachas et al. (2000), Richaud et al. (2000) et Wondimu et al. (2000) ont utilisé la méthode sur différents produits organiques de la chaîne pétrolière et avec des masses oscillant entre 3 et $250 \mathrm{mg}$.

Une bonne mise au point de la minéralisation par microonde est faite par Wondimu et al. (2000). 24 éléments sont dosés en mode aqueux après minéralisation de $250 \mathrm{mg}$. Parmi les paramètres optimisés, une attention particulière est portée aux étapes de la minéralisation (puissance et temps appliqué), au suivi de la pression à l'intérieur des bombes, ainsi qu'au mélange avec des réactifs oxydants et acides. Une évaporation finale jusqu'à un volume de $2 \mathrm{ml}$ permet de réduire la concentration des acides dans le minéralisat final et de diluer le tout dans un minimum de $25 \mathrm{ml}$ d'eau distillée. Cette dernière étape est importante puisqu'elle permet de contrecarrer le désavantage majeur de la technique de minéralisation: une dilution importante, inhérente à la forte concentration d'acide dans le minéralisat final. Cette dilution abaisse notablement les limites de détection dans la matrice pétrolière et rend par conséquent cette pratique peu avantageuse au regard de l'introduction directe de produits pétroliers en ICP-MS.

Dans un autre registre, mais toujours dans le domaine pétrolier, une application récente de l'ICP-MS pour l'analyse de liquide ionique soluble en milieu aqueux a récemment été publiée. Ainsi McCamley et al. (2004) ont utilisé un ICP-MS haute résolution (ICP-HRMS) pour mesurer les impuretés de chlore résiduel après synthèse dans 6 liquides ioniques issus de voies de synthèse différentes. Avec une limite de quantification de $10 \mu \mathrm{g} / \mathrm{l}$, le gain en sensibilité de l'ICP-HRMS au regard des techniques de type électrophorèse capillaire est de 100 fois. Une des limitations de la méthode proposée est l'obligation actuelle d'avoir des liquides ioniques solubles dans l'eau, mais les travaux vus ci-dessus en milieu organique laissent entrevoir de prochaines issues sur des liquides ioniques solubles en milieu organique.

\section{Applications récentes en spectrométrie de masse par plasma à couplage inductif et dilution isotopique dans le domaine pétrolier}

À l'exception de quelques éléments qui sont mono-isotopiques ( $\mathrm{Na}, \mathrm{Al}, \mathrm{Co}$, As, etc.), les éléments du tableau périodique présentent plusieurs isotopes stables. La dilution isotopique est illustrée par la détermination du nombre total d'atomes $\mathrm{N}_{\mathrm{x}}$ d'un élément présentant au moins deux isotopes $\mathrm{A}$ et $\mathrm{B}$. Si une quantité connue de ce même élément est ajouté à l'échantillon, mais de répartition isotopique $\mathrm{N}_{\mathrm{a}} / \mathrm{N}_{\mathrm{b}}$ différente de celle présente dans l'échantillon initial, le rapport isotopique dans le mélange entre l'ajout et l'échantillon est alors équivalent à $\left(\mathrm{N}_{\mathrm{a}} / \mathrm{N}_{\mathrm{b}}\right)_{\text {mélange }}$. Ce rapport isotopique peut être mesuré par ICP-MS et la concentration de l'élément N à doser calculée (Stumpf et Labarraque, 2003).

Cette méthode est considérée comme absolue car ne nécessitant pas d'étalonnage préalable de l'appareil. Les effets de matrice sont également limités puisque la détermination de la concentration se fait par des opérations effectuées sur l'échantillon lui-même. Ainsi l'incertitude sur le résultat obtenu est souvent voisin de quelques pourcents relatifs (1 à $5 \%$ ) quelque soit la concentration mesurée (Stumpf et Labarraque, 2003). Cette méthode est donc particulièrement adaptée à la mesure de traces. La principale difficulté réside dans l'acquisition d'étalons isotopiques, encore peu disponibles sur le marché et onéreux, et par le fait que la technique requiert habituellement un système capable de lever une grosse partie des interférences isobariques ou polyatomiques. Finalement, le biais de masse doit être connue dans chaque appareil afin de ne pas introduire d'erreur dans la mesure du rapport isotopique. Un appareil de type ICP-MS quadripolaire (ICP-QMS) équipé d'une cellule de collision ou un ICP-HRMS est donc souvent nécessaire. Ces deux types d'appareils commencent à faire leur apparition dans le domaine des analyses de produits pétroliers, surtout dans le domaine du dosage du S. Ils ont comme avantage de pouvoir lever les interférences poly-atomiques (ICP-QMS + cellule de collision) ou isobariques (ICP-HRMS) qui sont particulièrement présentes lors de l'introduction de produits pétroliers dans le plasma.

Evans et al. (2001) a déterminé la concentration en S de deux échantillons d'essence et de gasoil utilisé comme référence pour des essais inter-laboratoires européens.

Le soufre est présent avec les isotopes ${ }^{32} \mathrm{~S},{ }^{33} \mathrm{~S},{ }^{34} \mathrm{~S}$ et ${ }^{36} \mathrm{~S}$ avec des abondances naturelles de 95,02, 0,75, 4,21 et 0,02\% respectivement. Une des principales difficultés de ce dosage est l'interférence isobarique de l'oxygène sur les deux principaux isotopes $\left({ }^{16} \mathrm{O}_{2}\right.$ pour ${ }^{32} \mathrm{~S}$ et ${ }^{16} \mathrm{O}^{18} \mathrm{O}$ pour $\left.{ }^{34} \mathrm{~S}\right)$. Ces deux interférences sont facilement levées avec un ICP-HRMS.

Dans le cas d'Evans et al. (2001), le biais de masse instrumental est levé en mesurant le ratio isotopique du $\mathrm{Si}$ entre ${ }^{30} \mathrm{Si}$ et ${ }^{28} \mathrm{Si}$ qui est proche du ratio ${ }^{34} \mathrm{~S} /{ }^{32} \mathrm{~S}$ en terme de valeur mais également peu éloigné en terme de masse (4 u.m.a) et qui a l'avantage d'avoir peu de variations naturelles. 200 milligrammes de produits ont été minéralisés en bombe de quartz fermées dans un four micro-onde et dilués jusqu'à l'obtention de teneurs situées entre 100 et 200 ppb de S (dilution 50 à 300 fois). Les valeurs obtenues ont été comparées aux résultats obtenus sur un essai circulaire européen et montre l'excellente incertitude sur la mesure en comparaison des techniques mises en place lors de cet essai (fluorescence$\mathrm{X}$ dispersive en longueur d'onde et en énergie, fluorescence $\mathrm{UV}$ et $\mu$-coulométrie).

Heilmann et al. (2004) utilise également un ICP-HRMS pour déterminer le $\mathrm{S}$ dans les essences et gasoils par dilution isotopique. Le mode de préparation des échantillons est basé sur deux approches avec une microémulsion introduite par 
DIHEN et la minéralisation des produits organiques en bombe téflon avant dosage en aqueux. Il est montré dans cet article que la position du DIHEN dans la torche à plasma est cruciale pour la mesure en dilution isotopique et qu'un déplacement de 0,3 mm entraîne une baisse notable du signal de l'ion ${ }^{32} \mathrm{~S}$, provoquant un biais de masse plus important entre ${ }^{34} \mathrm{~S}$ et ${ }^{32} \mathrm{~S}$.

L'usage d'un ICP-MS quadrupolaire reste cependant possible pour le dosage du soufre et de ses isotopes dans les produits pétroliers comme l'a montré Yu et al. (2001). Pour contrecarrer l'interférence isobarique de l'oxygène sur les deux principaux isotopes, Yu et al. (2001) a introduit l'échantillon par l'intermédiaire d'une vaporisation électrothermique (ETV-ICP-MS) et en utilisant un mélange $\mathrm{N}_{2}$-Ar. Cette technique d'introduction a pour effet de limiter l'entrée de solvant dans la torche organique, limitant d'autant les dépôts carbonés sur les cônes d'échantillonnage. L'usage d'un mélange $\mathrm{N}_{2}$-Ar permet de piéger les traces d'oxygène résiduelles dans le plasma abaissant le nombre de coups sur le pic 32 de 10000 coups/seconde à 1600 coups/seconde pour $5 \%$ d'azote ajouté à l'argon. Le rapport ${ }^{32} \mathrm{~S} /{ }^{34} \mathrm{~S}$ est ainsi déterminé de façon aussi fiable qu'en TIMS, bien que les teneurs en soufre mesurées dans cet article soient très fortes (env. 8000 ppm de S).

\section{Applications récentes dans le domaine pétrolier en couplage chromatographique avec spectrométrie de masse par plasma à couplage inductif}

L'utilisation de l'ICP-MS en tant que méthode spectrométrique de détection couplée à une méthode de séparation en amont se démocratise de plus en plus. Plusieurs constructeurs proposent déjà à leur catalogue des ensembles complets comprenant moyens de séparation et ICP-MS déjà couplés. On retrouve donc également dans la littérature toutes les grandes méthodes de séparation couplées avec les différentes versions de l'ICP-MS.

Le couplage entre chromatographie en phase gazeuse et ICP-MS (GC-ICP-MS) est celui qui est le plus répandu et qui a démarré le plus tôt avec des publications dans le domaine pétrolier datant de 1992 avec l'analyse du plomb organique dans les essences (Kim et al., 1992). Depuis, Ebdon et al. (1994) a analysé le Co, Ni, Mn, Fe, Cu, Cr et Zn associé aux géo-porphyrines dans un extrait d'huile brute, l'aspect qualitatif est effectué en GC-MS alors que l'ICP-MS couplé à la chromatographie liquide (HPLC-ICP-MS) est préféré pour un dosage quantitatif.

Tao et al. (1998) a déterminé la spéciation du Hg dans les condensats de gaz. Sur 9 condensats analysés, deux contiennent majoritairement des formes organo-métalliques, 4 contiennent majoritairement des formes minérales entre $\mathrm{Hg}^{0}$ et $\mathrm{Hg}^{2+}$ et 3 contiennent un mélange de composés organo-métalliques et formes minérales.

Récemment, l'IFP s'est engagé dans un travail de spéciation du $\mathrm{Hg}$ et de l'As dans les condensats de gaz (Bouyssière,
2002). Les résultats obtenus sur 13 produits pétroliers pour la spéciation du $\mathrm{Hg}$ montrent que l'élément analysé est majoritairement sous forme d' $\mathrm{Hg}^{2+}$, et que le reste du $\mathrm{Hg}$ (env. $30 \%$ ) est de forme $\mathrm{Hg}^{0}$. Pour l'arsenic, il a été montré que les formes de l'As sont volatiles et donc dosables en direct par GC-ICP-MS sans dérivatisation initiale et évoluent au cours du temps pendant le stockage du produit.

Finalement, le couplage entre GC et ICP-MS a été utilisé pour améliorer (Bouyssière et al., 2004) la sensibilité du dosage du $\mathrm{S}$ après séparation sur colonne chromatographique. La limite de détection atteinte est cent fois meilleure qu'avec les détecteurs dédiés au soufre démontrant par là la puissance de l'ICP-MS utilisé en tant que détecteur derrière une méthode de séparation.

La chromatographie liquide a aussi été utilisée avec l'ICPMS en tant que système de détection, mais les applications dans le domaine pétrolier se limite à la séparation de métalloou géo-porphyrines déjà extraites de produit pétrolier ou issues d'autres milieux (Kumar et al., 1994). L'existence de géo-porphyrines (Ebdon et al., 1994) complexée avec du Ti est révélée par cette technique alors que ce complexe n'avait jusqu'alors jamais été observé.

Dans le cas des couplages déjà mentionnés, l'utilisation d'une cellule de collision avant le détecteur permet de lever certaines interférences limitant le dosage. C'est le cas de l'étude de Bouyssière et al. (2004) qui utilisent de l'hélium pour éliminer l'interférence de ${ }^{16} \mathrm{O}_{2}$ sur le ${ }^{32} \mathrm{~S}$. L'oxygène est en revanche utilisé par Hann et al. (2004) pour déplacer la détection du $\mathrm{S}$ sur le pic ${ }^{32} \mathrm{~S}^{16} \mathrm{O}$ localisé dans une zone sans interférence.

Finalement, l'électrophorèse capillaire a aussi trouvé ses applications avec l'usage d'un ICP-MS en tant que détecteur. Aucune application n'est répertoriée dans le domaine pétrolier à ce jour et la séparation de métallo-porphyrines effectués par Ackley et al. (2000) est l'exemple le plus proche des préoccupations du milieu pétrolier.

\section{Applications récentes en ablation laser couplée à la spectrométrie de masse par plasma à couplage inductif sur produits pétroliers}

L'ablation laser dans le domaine des produits pétroliers commence à être utilisée pour des essais ponctuels sur certains produits. Ainsi, Bings (2002) a utilisé l'ablation laser couplée avec un ICP-MS pour déterminer 11 métaux (mesure de 19 isotopes) dans les huiles lubrifiantes. Deux calibrations différentes ont été utilisées :

- un étalonnage externe en ablatant des solutions organiques de concentrations connues fabriquées à partir d'étalons «Conostan »;

- un étalonnage «interne» en ablatant de l'huile blanche tout en nébulisant dans le flux d'Ar en amont de la chambre d'ablation des solutions aqueuses contenant les éléments à analyser. 
Les limites de détection sont données dans le tableau 6 cidessous.

Les résultats obtenus avec les deux calibrations sont comparés avec les résultats obtenus après minéralisation microonde et une très bonne concordance est montrée.

Booth et al. (1999) utilise l'ablation laser pour doser les éléments traces dans le charbon et calibrent leur appareil à l'aide de matériaux certifiés en charbon. Les charbons sont pressés avec un liant commercial sous forme de pastille de $32 \mathrm{~mm}$ de diamètre. D'autres charbons sont analysés et les résultats comparés soit aux teneurs de référence, soit à ceux obtenus par d'autres auteurs. Une bonne cohérence entre toutes ces valeurs est montrée. Les limites de détections obtenues dans ce travail sont également données dans le tableau 6.

Une étude de Mossman et al. (2001) sur les inclusions de bitume dans des dépôts miniers utilise les propriétés qualitatives de l'ablation laser couplée avec un ICP-MS pour déterminer si les éléments traces observés dans ces dépôts sont plutôt associés aux bitumes ou aux différentes phases constituant la roche alentour. La calibration de l'appareil est effectuée en ablatant directement une solution organique multi-élémentaire diluée dans l'huile blanche selon le principe proposé par Günther et al. (1997).

\section{CONCLUSION}

Les besoins actuels et à venir en détermination de teneurs traces de métaux dans les produits pétroliers semblent donc pouvoir être satisfaits à la vue des travaux passés en revue avec les trois techniques que sont le GFAAS, l'ICP-OES et l'ICP-MS. Les caractéristiques globales des différentes techniques sont reprises dans le tableau 7.

L'absorption atomique four est une technique mature avec des procédures de mesures bien décrites dans la littérature. Son application est facile à tout type de produits pétroliers, liquides ou diluables dans un solvant injectable dans le four graphite. Malgré une période de vie qui commence à être longue, l'absorption atomique four continue de susciter de l'intérêt, non plus en développements de la technique analytique, mais en applications développées vis-à-vis de problématiques concrètes.

Si les montées en température du four permettent justement l'injection de produits volatiles, les paliers et programmes en température restent spécifiques à un ou plusieurs éléments, limitant d'autant les mesures multi-éléments souvent souhaitées dans l'industrie pétrolière.

Les techniques ICP prennent alors le relais, avec la spectrométrie optique qui est à ce jour bien utilisée dans les laboratoires en routine avec des conditions d'injection des produits souvent bien maîtrisées. Les travaux de développement observés restent dans le domaine de l'introduction de l'échantillon, avec surtout des travaux portés sur la nébulisation ultrasonique et l'utilisation après cela d'une membrane de désolvatation. Si un point devrait être retenu entre les travaux publiés et l'utilisation quotidienne qu'en font bon nombre de laboratoires, ce serait l'ajout de l'oxygène dans le

TABLEAU 6

Limites de détection par ablation laser et ICP-MS

Detection limit obtained with LA-ICP-MS

\begin{tabular}{|c|c|c|c|c|c|c|c|c|c|c|c|c|c|}
\hline Isotopes & ${ }^{23} \mathrm{Na}$ & ${ }^{26} \mathrm{Mg}$ & ${ }^{27} \mathrm{Al}$ & ${ }^{48} \mathrm{Ti}$ & ${ }^{52} \mathrm{Cr}$ & ${ }^{56} \mathrm{Fe}$ & ${ }^{59} \mathrm{Co}$ & ${ }^{60} \mathrm{Ni}$ & ${ }^{63} \mathrm{Cu}$ & ${ }^{75} \mathrm{As}$ & ${ }^{98} \mathrm{Mo}$ & ${ }^{109} \mathrm{Ag}$ & ${ }^{208} \mathrm{~Pb}$ \\
\hline $\begin{array}{l}\text { Bings (2002) } \\
\text { huile lubrifiante, } \mu \mathrm{g} / \mathrm{kg}\end{array}$ & 4 & 26 & 5 & 18 & 28 & 22 & 2 & 12 & 3 & - & - & 2 & 0,5 \\
\hline $\begin{array}{l}\text { Booth et al. (1999) } \\
\text { charbon, } \mu \mathrm{g} / \mathrm{kg}\end{array}$ & - & - & - & - & 13,2 & - & - & 6,9 & - & 13,2 & 1,7 & - & 4,4 \\
\hline
\end{tabular}

TABLEAU 7

Caractéristiques générales des techniques

General overview of the different techniques

\begin{tabular}{l|c|c|c|c|c}
\hline & GFAAS & ICP-OES & ICP-QMS & $\begin{array}{c}\text { ICP-QMS } \\
\text { + cellule de collision }\end{array}$ & ICP-HRMS \\
\hline Temps d'analyse & $\sim 1$ minute & $\begin{array}{c}10 \text { secondes } \\
-1 \text { minute }\end{array}$ & $1-2$ minutes & $1-2$ minutes & $2-5$ minutes \\
\hline Coût de l'appareil & $40-60 \mathrm{k} €$ & $80-100 \mathrm{k} €$ & $100-150 \mathrm{k} €$ & $150-200 \mathrm{k} €$ & $300-500 \mathrm{k} €$ \\
\hline Limite de détection & $1-10 \mathrm{ppb}$ & $0,5-10 \mathrm{ppm}$ & $0,1-10 \mathrm{ppb}$ & $0,1-10 \mathrm{ppb}$ & $0,05-5 \mathrm{ppb}$ \\
\hline
\end{tabular}


plasma que l'on retrouve dans toutes les publications récentes, et le fait que cette possibilité n'est pas offerte en version de base des ICP optiques vendus par les différents constructeurs. Ce dernier semble en effet améliorer la sensibilité des mesures en limitant les composés organiques imbrûlés dans le plasma, sources d'un bruit de fond non négligeable.

Finalement l'ICP-MS est en train d'émerger dans le milieu pétrolier, avec des applications qui restent encore dans le domaine de la recherche.

Les perspectives ouvertes avec ce nouvel outil semblent en revanche très prometteuses. D'une part les limites de quantification obtenues dans les travaux menés permettent d'atteindre un seuil que même l'absorption atomique four ne pouvait laisser espérer. Ces mêmes limites de quantification associées à plusieurs modes d'échantillonnage (ETV, ablation laser, etc.) permettent d'élargir la palette des produits analysables en ICP tout en maintenant une sensibilité importante.

Sur un autre registre, il est maintenant admis que les métaux présents dans les produits pétroliers sont sous différentes formes, mais peu d'études hormis celles sur le $\mathrm{S}, \mathrm{Hg}$, $\mathrm{Ni}$ et le $\mathrm{V}$ ont encore été entreprises pour définir les formes les plus génantes pour les mécanismes de réaction de raffinage et pétrochimie ou toxiques pour l'environnement. $\mathrm{Ce}$ n'est souvent qu'après l'arrêt très coûteux d'unités de raffinage que l'origine de l'empoisonnement du catalyseur de réaction est recherchée.

Si la compréhension de ces mécanismes est à ce jour bien développée dans les milieux de l'environnement, avec des spécifications ou limites de rejets associées à certaines formes de métaux, ce stade de compréhension n'est pas encore atteint dans le milieu pétrolier. C'est ce dernier verrou que l'ICP-MS couplé à différentes techniques de séparation pourra débloquer dans le futur. Le fait de voir apparaître depuis moins d'une année, sur le marché des appareils analytiques, des propositions «clef en main » permettant la spéciation d'espèces métalliques dans le milieu pétrolier, montre tout l'intérêt que l'industrie pétrolière commence à porter à ce sujet.

Finalement, les informations isotopiques que permet de collecter l'ICP-MS pourraient susciter pour l'amont pétrolier une source nouvelle de compréhension des mécanismes de genèse et de transport des produits pétroliers au sein de la croûte terrestre.

\section{RÉFÉRENCES}

Ackley, K.L., Day, J.A. et Caruso, J.A. (2000) Separation of Metalloporphyrins by Capillary Electrophoresis with UV Detection and Inductively Coupled Plasma Mass Spectrometric Detection. J. Chromatography A, 888, 293-298.

Al-Swaidan, H.M. (1996) The Determination of Lead, Nickel and Vanadium in Saudi Arabian Crude Oil by Sequential Injection Analysis/Inductively-Coupled Plasma Mass Spectrometry. Talanta, 43, 1313-9.
Al-Swaidan, H.M. et Al-Gadi, A.A. (2002) Detection of Lead and Vanadium as Trace Pollutants in Saudi Arabian Petroleum and its Products by SIA/ICP/MS. J. Saudi Chem. Soc., 1, 1-4.

Alves, L.C., Minnich, M.G., Wiederin, D.R. et Houk R.S. (1994) Removal of Organic Solvents by Cryogenic Desolvatation in Inductively Coupled Plasma Mass Spectrometry. JAAS, 9, 399403.

Aucelio, R.Q. et Curtius, A.J. (2002) Evaluation of Electrothermal Atomic Absorption Spectrometry for Trace Determination of Sb, As and $\mathrm{Se}$ in Gasoline and Kerosene Using Microemulsion Sample Introduction and Two Approaches for Chemical Modification. JAAS, 17, 242-7.

Azeredo Amaro, J.A. et Ferreira S.L.C. (2004) Application of Factorial Design and Doehlert Matrix in the Optimisation of Instrumental Parameters for Direct Determination of Silicon in Naphta Using Graphite Furnace Atomic Absorption Spectrometry. $J A A S, 19,246-9$.

Begak, O.Y. et Sytoezhko, A.M. (2004) Identification of Oxygencontaining Compounds in Benzine and Kerosene Oil Fractions and Determination of Sulfur and Halogen Content in them. Russian Journal of Applied Chemistry, 4, 653-6.

Bings, N.H. (2002) Direct Determination of Metals in Lubricating Oils by Laser Ablation Coupled to Inductively Coupled Plasma Time-of-flight Mass Spectrometry. JAAS, 17, 759-67.

Bing-Quan, Z., Jing-Lian, Z., Xiang-Lin, T., Xiang-Yang, C., Cai-Yuan, F., Ying, L. et Ju-Ying, L. (2001) Pb and Nd Isotopic Features in Organic Matter from China and their Implication for Petroleum Generation and Migration. Geochim. Cosmochim. Acta, 65, 15, 2555-70.

Booth, C.A., Spears, D.A., Krause, P. et Cox, A.G. (1999) The Determination of Low Level Trace Elements in Coals by Laser Ablation-Inductively Coupled Plasma-Mass Spectrometry. Fuel, 78, 1665-70.

Botto, R.I. (1993) Applications of Ultrasonic Nebulization in the Analysis of Petroleum and Petrochemicals by Inductively Coupled Plasma Atomic Emission Spectrometry. JAAS, 8, 51-7.

Botto, R.I. et Zhu, J.J. (1994) Use of an Ultrasonic Nebulizer with Membrane Desolvation for Analysis of Volatile Solvents by Inductively Coupled Plasma Atomic Emission Spectrometry. JAAS, 9, 905-12.

Botto, R.I. et Zhu, J.J. (1996) Universal Calibration for Analysis of Organic Solutions by Inductively Coupled Plasma Atomic Emission Spectrometry. JAAS, 11, 675-81.

Botto, R.I. (2002) Trace Element Analysis of Petroleum Naphtas and Tars Using Direct Injection ICP-MS. Canadian Journal of Analytical Sciences and Spectroscopy, 47, 1, 1-13.

Bouyssiere, B. (2002) Spéciation du mercure et de l'arsenic dans les condensats de gaz. Thèse, Université de Pau et des Pays de l'Adour.

Bouyssiere, B., Leonhard, P., Pröfrock, D., Baco, F. et LopezGarcia, C. (2004) Speciation of Sulfur in Petroleum Products by Capillary Gas Chromatography with ICP-Collision Cell-MS Detection. JAAS, 19, 5, 700-2.

Brenner, I.B., Zander, A., Plantz, M. et Zhu, J. (1997) Characterization of an Ultrasonic Nebulizer-Membrane Separation Interface with Inductively Coupled Plasma Mass Spectrometry for the Determination of Trace Elements by Solvent Extraction. JAAS, 12, 273-9.

Caldiero, L., Anelli, M.A., Nodari, R. et Pollesel, P. (1997) Metals Determination by Microwaves Digestion and ICP-AES Technique of Upgraded Liquids from Heavy Petroleum Residus. Preprints of Papers - American Chemical Society, Division of Fuels Chemistry, 43, 2, 942-6.

Cassella, R.J., Barbosa, B.A.R.S., Santelli, R.E. et Rangel, A.T. (2004) Direct Determination of Arsenic and Antimony in Naphta 
by Electrothermal Atomic Absorption Spectrometry with Microemulsion Sample Introduction and Iridium Permanent Modifier. Anal. Bioanal. Chem., 379, 66-71.

Dos Santos, W.N.L., Dias, F.S, Fernandes, M.S., Reboucas, M.V., Vale, M.G.R., Welz, B. et Ferreira; S.L.C. (2005) Application of Multivariate Technique in Method Development for the Direct Determination of Copper in Petroleum Condensate Using Graphite Furnace Atomic Absorption Spectrometry. JAAS, 20, 127-9.

Dreyfus, S., Pécheyran, C., Magnier, C., Prinzhofer, A., Lienemann, C.P. et Donard, O.F.X. (2005) Direct Trace and Ultra-Trace Metals Determination in Crude Oil and Fractions by Inductively Coupled Plasma Mass Spectrometry. ASTM Journal, 2, 9, 1-8.

Druon, M., Mondon, V. et Touzé, P. (2000) Introduction de produits organiques en spectrométrie ICP-AES par simple mise en émulsion. Applications au dosage de Ni et V dans les bitumes. Bulletin des laboratoires des Ponts et chaussées, 225, 13-20.

Duyck, C., Miekeley, N., Porto da Silveira, C.L. et Szatmari, P. (2003) Trace Element Determination in Crude Oil and its Fractions by Inductively Coupled Plasma Mass Spectrometry Using Ultrasonic Nebulization of Toluene Solutions. Spectrochim. Acta Part B, 57, 12, 1979-90.

Ebdon, L., Evans, E.H., Pretorius, W.G. et Rowland, S. J. (1994) Analysis of Geoporphyrins by High-temperature Gas Chromatography Inductively Coupled Plasma Mass Spectrometry and High-performance Liquid Chromatography Inductively Coupled Plasma Mass Spectrometry. JAAS, 9, 939-43.

Edlund, M., Visser, H. et Heitland, P. (2002) Analysis of Biodiesel by Argon-oxygen Mixed-gas Inductively Coupled Plasma Optical Emission Spectroscopy. JAAS, 17, 232-5.

Escobar, M.P., Smith, B.W. et Winefordner, J.D. (1996) Determination of Metallo-Organic Species in Lubricating Oil by Electrothermal Vaporization Inductively Coupled Plasma Mass Spectrometry. Anal. Chim. Acta, 320, 11-7.

Evans, P., Wolff-Briche, C. et Fairman, B. (2001) High Accuracy Analysis of Low Level Sulfur in Diesel Fuel by Isotope Dilution High Resolution ICP-MS, Using Silicon for Mass bias Correction of Natural Isotope Ratios. JAAS, 16, 964-9.

Günther, D.R., Frischknecht, R., Müschenborn, H.J. et Heinrich, C.A. (1997) Direct Liquid Ablation: A New Calibration Strategy for Laser Ablation-ICP-MS Microanalysis of Solids and Liquids. Fresenius J. Anal. Chem., 359, 390-3.

Hann, S., Koellensperger, G., Obinger, C., Furtmüller, P.G. et Stingeder, G. (2004) SEC-ICP-DRCMS and SEC-ICP-SFMS for Determination of Metal-sulfur Ratios in Metalloproteins. JAAS, 19, 74-9.

Heilmann, J., Boulyga, S.F. et Heumann, K.G.(2004) Accurate Determination of Sulfur in Gasoline and Related Fuel Samples Using Isotope Dilution with Direct Sample Injection and Microvawe-Assisted Digestion. Anal. Bioanal. Chem., 380, 190-7.

Kahen, K., Strubinger, A., Chirinos, J.R. et Montaser, A. (2003) Direct Injection Efficiency Nebulizer-inductively Coupled Plasma Mass Spectrometry for Analysis of Petroleum Samples. Spectrochim. Acta Part B, 58, 397-413.

Kim, A.W., Hill, S.J. et Rowland, S.J. (1992) Determination of Organometallic Compounds by Capillary Gas Chromatographyinductively Coupled Plasma Mass Spectrometry. J. High Res. Chromatogr., 15, 665-8.

Krengel-Rothensee, K., Richter, U. et Heitland, P. (1999) LowLevel Determination of Non-metals $(\mathrm{Cl}, \mathrm{Br}, \mathrm{I}, \mathrm{S}, \mathrm{P})$ in Waste Oils by Inductively Coupled Plasma Optical Emission Spectrometry Using Prominent Spectral Lines in the 130-190 nm. JAAS, 14, 699-702.

Kumar, S.J. et Gangadharan, S. (1999) Determination of Trace Elements in Naphta by Inductively Coupled Plasma Spectrometry Using Water-in-Oil Emulsions. JAAS, 14, 967-71.
Kumar, U., Dorsey, J.G. et Caruso, J.A. (1994) Metalloporphyrin Speciation by Liquid Chromatography and Inductively Coupled Plasma-mass Spectrometry. J. Chromato. Science, 32, 282-5.

Lachas, H., Richaud, R., Herod, A.A., Dugwell, D.R. et Kandiyoti, R. (2000) Determination of Trace Element by Inductively Coupled Plasma Mass Spectrometry of Biomass and Fuel Oil Reference Materials Using Milligram Sample Sizes. Rapid Communications in mass spectrometry, 14, 5, 335-43.

Mbileni, C.N., Ngobeni, P., Katskov, D.A. et Panichev, N. (2002) Determination of Lead and Cadmium in Organic Solutions by Electrothermal Atomic Absorption Spectrometry with a Transverse Heated Filter Atomizer. JAAS, 17, 236-41.

McCamley, K., Warner, N.A., Lamoureux, M.M., Scammells, P.J. et Singer, R.D. (2004) Quantification of Chloride Ion Impurities in Ionic Liquids Using ICP-MS Analysis. Green Chemistry, 6, 341-4.

Mossman, D.J, Jackson, S. E. et Gauthier-Lafaye, F. (2001) Trace Element and Isotopic Analysis by Laser Ablation ICP-MS of Ore Deposit bitumens: A Test Case with Uranium Ores from Oklo, Gabon. Energy Sources, 23, 809-22.

Nakamoto, Y., Ishimaru, T., Endo, N. et Matsusaki, K. (2004) Determination of Vanadium in Heavy Oils by Atomic Absorption Spectrometry Using a Graphite Furnace Coated with Tungsten, Analytical Sciences, 20, 739-41.

de Oliveira, A.P., de Moraes, M., Gomes Neto, J.A. et Lima, E.C. (2002) Simultaneous Determination of $\mathrm{Al}, \mathrm{As}, \mathrm{Cu}, \mathrm{Fe}$ and $\mathrm{Ni}$ in Fuel Ethanol by GFAAS. Atomic Spectroscopy, 23, 2, 39-43.

de Oliveira, A.P., de Moraes, M., Gomes Neto, J.A. et Lima, E.C. (2002) Direct Determination of Al, As, Cu, Fe, Mn and Ni in Fuel Ethanol by Simultaneous GFAAS Using Integrated Platforms Pretreated with W-Rh Permanent Modifier Together with Pd+Mg Modifier. Atomic Spectroscopy, 23, 6, 190-5.

Olsen, S.D., Filby, R.H., Brekke, T. et Isaksen, G.H. (1995) Determination of Trace Elements in Petroleum Exploration Samples by Inductively Coupled Plasma Mass Spectrometry and Instrumental Neutron Activation Analysis. Analyst, 120, 1379-90.

Olsen, S., Westerlund, S. et Visser, R.G. (1997) Analysis of Metals in Condensates and Naphta by Inductively Coupled Plasma Mass Spectrometry. Analyst, 122, 1229-34.

Reboucas, M.V., Ferreira, S.L.C. et de Barros Neto, B. (2003) Arsenic Determination in Naphta by Electrothermal Atomic Absorption Spectrometry after Preconcentration Using Multiple Injections. $J A A S, \mathbf{1 8}, 1267-73$.

Resano, M., Garcia-Ruiz, E., Crespo, C., Vanhaecke, F. et Belarra, M.A. (2003) Solid Sampling-Graphite Furnace Atomic Absorption Spectrometry for Palladium Determination at Trace and Ultratrace Levels. JAAS, 18, 1477-84.

Richaud, R., Lachas, H., Lazaro, M.J., Clarke, L.J., Jarvis, K.E., Herod, A.A., Gibb, T.C. et Kandiyoti, R. (2000) Trace Elements in Coal Derived Liquids: Analysis by ICP-MS and Mössbauer Spectroscopy. Fuel, 79, 57-67.

Saint'Pierre, T., Dias, L. F., Pozebon, D., Aucelio, R.Q., Curtius, A.J. et Welz, B. (2002) Determination of $\mathrm{Cu}, \mathrm{Mn}, \mathrm{Ni}$ and $\mathrm{Sn}$ in Gasoline by Electrothermal Vaporization Inductively Coupled Plasma Mass Spectrometry, and Emulsion Sample Introduction. Spectrochim Acta Part B, 57/12, 1991-2001.

Souza, R.M., Da Silveira, C.L.P. et Aucelio, R.Q. (2004) Determination of Refractory Elements in Used Lubricating Oil by ICPOES Employing Emulsified Sample Introduction and Calibration with Inorganic Standards. Analytical Sciences, 20, 351-5.

Stumpf, C. et Labarraque, G. (2003) La dilution isotopique par ICP/MS : une méthode de référence pour l'analyse d'éléments traces. Spectra Analyse, 32, 234, 14-8.

Tao, H., Murakami, T., Tominaga, M. et Miyazaki, A. (1998) Mercury Speciation in Natural Gas Condensate by Gas 
Chromatography-Inductively Coupled Plasma Spectrometry. $J A A S$, 13, 1085-93.

Vale, M.G.R., Damin, I.C.F., Klassen, A., Silva, M.M., Welz, B., Silva, A.F., Lepri, F.G., Borges, D.L.G. et Heitmann, U. (2004) Method Development for the Determination of Nickel in Petroleum Using Line-source and High Resolution ContinuumSource Graphite Furnace Atomic Absorption Spectrometry. Microchemical Journal, 77, 131-40.

Wang, T., Jia, X. et Wu, J. (2003) Direct Determination of Metals in Organics by Inductively Coupled Plasma Atomic Emission Spectrometry in Aqueous Matrices. J. Pharma Biomedical Anal., 33, 639-46.
Wondimu, T., Goessler, W. et Irgolic, K.J. (2000) Microwave Digestion of Residual Fuel Oil for the Determination of Trace Elements by Inductively Coupled Plasma-mass Spectrometry. Fresenius Journal of Anal. Chem., 367/1, 35-42.

Yu, L.L., Kelly, W.R., Fassett, J.D. et Vocke, R.D. (2001) Determination of Sulfur in Fossil Fuels by Isotope Dilution Electrothermal Vaporization Inductively Coupled Plasma Mass Spectrometry. JAAS, 16, 140-5.

Manuscrit définitif remis en juillet 2005 or distributed for profit or commercial advantage and that copies bear this notice and the full citation on the first page. Copyrights for components of this work owned by others than IFP must be honored. Abstracting with credit is permitted. To copy otherwise, to republish, to post on servers, or to redistribute to lists, requires prior specific permission and/or a fee: Request permission from Documentation, Institut français du pétrole, fax. +33147527078 , or revueogst@ifp.fr. 